\title{
DEFORMATIONS OF RIEMANNIAN STRUCTURES
}

\author{
BY K. SRINIVASACHARYULU
}

Communicated by Raoul Bott, September 17, 1963

It has been shown in [1] that a Kählerian deformation of an irreducible compact Hermitian symmetric space $V$ is again symmetric and is isomorphic to it. The aim of this note is to prove this for any compact Hermitian symmetric space, irreducible or not. We first prove the following Theorem 1 which is a slight generalization ${ }^{1}$ of Theorem 2 in [1].

Theorem 1. Let $V$ and $B$ be two differentiable manifolds and let $R(t)$ be a riemannian structure on $V$ which depends in a differentiable way on $t \in B$; let $F$ be any compact riemannian manifold. Then the set of points $t \in B$ such that $(V, R(t))$ is isomorphic to $F$ is closed in the set of points $t \in B$ such that $(V, R(t))$ is complete.

Proof. Denote by $C$ the set of all points $t \in B$ such that $V_{t}=(V, R(t))$ is complete and let $t_{n}$ be a sequence of points in $C$ converging to a point $t_{0}$ such that (i) $t_{0} \in C$, and (ii) $V_{t_{n}}=\left(V, R\left(t_{n}\right)\right)$ is isomorphic to $F$ for all $n>0$. We prove that $V_{0}$ is isomorphic to $F$. Let $h_{n}$ be an isomorphism of $F$ onto $V_{t_{n}}=\left(V, R\left(t_{n}\right)\right)$ and let $r_{0}$ be an orthonormal frame in the tangent space $T_{0}(F)$ at $p_{0}$ of $F$; denote by $r_{t_{n}}$ the orthonormal frame $h_{n}\left(r_{0}\right)$ at $x_{n}=h_{n}\left(p_{0}\right)$ in $V_{t_{n}}$. Since $V_{0}$ is compact, the sequence of points $\left\{x_{n}\right\}$ has a limit point $x_{0}$. Since the set of all orthonormal frames at all points of a compact neighbourhood of $x_{0}$ in $V_{0}$ is again compact, the sequence $\left\{r_{t_{n}}\right\}$ admits a limit point $r_{0}^{\prime}$. Let $l_{t}$ be the linear mapping of $T_{x_{1}}\left(V_{t}\right)$ onto $T_{x_{n}}\left(V_{t_{n}}\right)$ which maps $r_{1}$ onto $r_{t_{n}}$; let $u$ be a unit vector at $x_{1}$ and let $y$ be the end-point of the geodesic arc of length $s$, of origin $x_{1}$ and tangent to $u$; then $y=\phi(s, u)$ where $\phi$ is a differentiable function of $u$ and of $s$. If $y_{t}$ is the end-point of the geodesic arc of origin $x_{t}$ and tangent to $l_{t}(u)$, we have $y_{t}$ $=\phi(s, u, t)$ where $\phi(s, u, 1)=\phi(s, u)$. For $t>0$, we have $y_{t}=h_{t}(y)$; as $t_{n} \rightarrow 0, h_{n}(u)$ tends to a vector $h_{0}(u)$ and $h_{n}(y)$ tends to a point $h_{0}(y)=\phi(s, u, 0)$. It is easy to see that $h_{0}$ is a well-defined differentiable mapping of $V_{1}$ into $V_{0}$; since $V_{0}$ is complete, it follows that $h_{0}$ is onto. We prove that $h_{0}$ is one-to-one; let $z_{1}$ and $z_{2}$ be two distinct points of $V_{1}$ and let $d_{t}$ denote the metric defined by $R(t)$ on $V_{t}$. Since

${ }^{1}$ This generalization of Theorem 2 in [1] has been suggested to me by Professor J. L. Koszul. The proof of Theorem 2 [1] has been suggested to me by Professor C. Ehresmann. This research is supported in part by NSF G-18834. 
$h_{t}$ is an isomorphism of $F$ onto $V_{t}$, we have $d_{t}\left(h_{t}\left(z_{1}\right), h_{t}\left(z_{2}\right)\right)=d_{1}\left(z_{1}, z_{2}\right)$. Suppose that $h_{0}\left(z_{1}\right)=h_{0}\left(z_{2}\right)$; we have

$$
d_{t}\left(h_{t}\left(z_{1}\right), h_{t}\left(z_{2}\right)\right)<d_{t}\left(h_{0}\left(z_{1}\right), h_{t}\left(z_{1}\right)\right)+d_{t}\left(h_{0}\left(z_{2}\right), h_{t}\left(z_{2}\right)\right) \text {. }
$$

Since $h_{t}\left(z_{1}\right)$ and $h_{t}\left(z_{2}\right)$ tend to $h_{0}\left(z_{1}\right)$ as $t \rightarrow 0$, we see that $d_{t}\left(h_{t}\left(z_{1}\right), h_{t}\left(z_{2}\right)\right)$ $=d_{1}\left(z_{1}, z_{2}\right)=0$, a contradiction. Thus to prove that $h_{0}$ is an isomorphism, it is sufficient to prove that $h_{0}$ is a local isomorphism. Let $U_{1}$ be a normal geodesic neighbourhood of $x_{1}$ in $V_{1}$; for each $z \in U_{1}$, there exists a unique geodesic $\gamma_{1}$ of origin $x_{1}$ and end-point $z=\phi(a, u) . h_{0}$ associates to each geodesic $\gamma_{1}$ ni $U_{1}$ a geodesic $\gamma_{0}$ of origin $x_{0}$ and to each normal coordinate system in $U_{1}$ a normal coordinate system in a neighbourhood $U_{0}$ of $x_{0}$ in $V_{0}$; hence the restriction of $h_{0}$ to $U_{1}$ is an isomorphism. Since $\gamma_{1}$ is compact, there exists a positive number $\epsilon$ such that there exists a geodesic coordinate system of center $z=\phi(a, u)$ in $V_{1}$ and of center $\phi(s, u, 0)=h_{0}(z)$ of radius $\epsilon$ for $0<s<a$. If $h_{0}$ is a local isomorphism in the neighbourhood of $z$ in $V_{1}$, the restriction of $h_{0}$ to a ball of radius $<\epsilon$ of center $z$ is an isomorphism; in fact, let $r_{1}^{\prime}$ be an orthonormal frame at $z$ in $V_{1}$ and let $h_{0}\left(r_{1}^{\prime}\right)$ be the corresponding frame in $V_{0}$; by the corresponding geodesic arcs of origins $z$ and $h_{0}(z)$, we can define as above, a differentiable mapping $h_{0}$ of $V_{1}$ onto $V_{0}$ and it is easy to see that $h_{0}^{\prime}=h_{0}$. Let $s_{1}$ be the upper bound of the values of $s$ for which $h_{0}$ is a local isomorphism of a neighbourhood of $\phi(s, u)$ in $V_{1}$ into $V_{0}$; then we can find an $s^{\prime}$ such that there exists a geodesic ball of center $\phi\left(s^{\prime}, u\right)$ which contains $\phi\left(s_{1}, u\right)$; by repeating the above argument, one can show that $h_{0}$ is a local isomorphism in a neighbourhood of $\phi\left(s_{1}, u\right)$, which leads to a contradiction. Hence $h_{0}$ is a local isomorphism in the neighbourhood of each point and the theorem follows.

A similar argument proves the following:

THEOREM 2. Let $V$ be a complex manifold and let $K(t)$ be a Kähler metric on $V$ depending in a differentiable way on a parameter $t \in B$, where $B$ is a differentiable manifold; let $F$ be a compact $K$ ähler manifold. Then the set of points $t \in B$ for which $(V, K(t))$ is isomorphic to $F$ is closed in the set of points $t \in B$ such that $(V, K(t))$ is complete.

Proof. We may assume that $h_{t}$ is an isomorphism (complex analytic) of $F$ onto $V_{t}$ and consider the unitary frames in the proof of the above theorem; then the mapping $\phi\left(x_{1}, u\right)$ is complex analytic. It follows, then, that $h_{0}$ is a complex analytic mapping of $V_{1}$ onto $V_{0}$ and it can be shown, as above, that $h_{0}$ is a local isomorphism.

As an application, we have the following:

Corollary. Any Kählerian deformation of a compact Hermitian 
symmetric space $V$ (irreducible or not) is again Hermitian symmetric and is isomorphic to $V$.

Proof. Since $H^{1}(V, \theta)=0$ [2], we see that the set of points $t \in B$ for which $V_{t}$ is isomorphic to $V$ is an open set in $B$ [3]; it is also closed by Theorem 2 .

\title{
REFERENCES
}

1. K. Srinivasacharyulu, Thesis to appear in Séminaire Ehresmann, Paris.

2. R. Bott, Homogeneous vector bundles, Ann. of Math. (2) 66 (1957), 203-248.

3. K. Kodaira and D. C. Spencer, On deformations of complex structures. I, II, Ann. of Math. (2) 67 (1958), 328-465.

UNIVERSITY OF MARYLAND AND

INSTITUTE FOR ADVANCED STUdy

\section{ON THE BEST APPROXIMATION FOR SINGULAR IN- TEGRALS BY LAPLACE-TRANSFORM METHODS}

\author{
BY HUBERT BERENS AND P. L. BUTZER \\ Communicated by Felix Browder, October 9, 1963
}

1. Introduction. Let $f(t)$ be a Lebesgue-integrable function in $(0, R)$ for every positive $R$. We denote by

$$
J_{\rho}(t)=\rho \int_{0}^{t} f(t-u) k(\rho u) d u
$$

a general singular integral with parameter $\rho>0$ and kernel $k$ having the following property $(\mathrm{P}): k(u) \geqq 0$ in $0 \leqq u<\infty, k \in L(0, \infty)$, and $\int_{0}^{\infty} k(u) d u=1$.

If we restrict the class of functions $f(t)$ such that $e^{-c t} f \in L_{p}(0, \infty)$, $1 \leqq p<\infty$, for every $c>0$, and if $k$ satisfies $(\mathrm{P})$, then the following statements hold:

(i) $J_{\rho}(t)$ exists as a function of $t$ almost everywhere, $e^{-c t} J_{\rho}$ $\in L_{p}(0, \infty)$ for every $c>0$, and $\left\|e^{-c t} J_{\rho}\right\|_{L_{p}(0, \infty)} \leqq\left\|e^{-c t} f\right\|_{L_{p}(0, \infty)}$;

(ii) $\lim _{\rho} \uparrow_{\infty}\left\|e^{-c t}\left\{f-J_{\rho}\right\}\right\|_{p}=0$.

Furthermore, we denote by

$$
\hat{f}(s)=\int_{0}^{\infty} e^{-s t} f(t) d t \quad(s=\sigma+i \tau, \operatorname{Re} s>0)
$$

the Laplace-transformation of a function $f$ belonging to one of the classes described above, and the Laplace-Stieltjes-transform of a 